\title{
Spatial and temporal dynamics of Belousov-Zhabotinsky reaction: A STEM approach
}

\author{
J.A. Arzola-Flores \\ Instituto de Física, Benemérita Universidad Autónoma de Puebla, \\ Av. San Claudio S/N. Col. San Manuel, 72570, Puebla, Pue., México. \\ E. García-García and J. F. Rojas-Rodríguez \\ Facultad de Ciencias Físico Matemáticas, Benemérita Universidad Autónoma de Puebla, \\ Av. San Claudio S/N. Col. San Manuel, 72570, Puebla, Pue., México. \\ R. Murueta-Fortiz and G. Corona-Morales \\ Facultad de Ciencias de la Electrónica. Benemérita Universidad Autónoma de Puebla, \\ Av. San Claudio S/N. Col. San Manuel, 72570, Puebla, Pue., México. \\ A. Hernández-Santiago \\ Laboratorio de Química Biofísica, Facultad de Ciencias Químicas, Benemérita Universidad Autónoma de Puebla, \\ Av. San Claudio S/N. Col. San Manuel, 72570, Puebla, Pue., México. \\ E. Ayala-Herrera and E. Vidal-Robles \\ Facultad de Ingeniería Química, Benemérita Universidad Autónoma de Puebla, \\ Av. San Claudio S/N. Col. San Manuel, 72570, Puebla, Pue., México.
}

Received 24 March 2020; accepted 7 April 2020

\begin{abstract}
An integrative methodology for teaching science is proposed through the STEM methodology (Science-Technology-Engineering-Mathematics). The STEM methodology provides students the opportunity to combine knowledge in an interdisciplinary and collaborative manner, allowing the development of creative and systemic thinking. As a model, we propose the experimental reproduction of the Belousov-Zhabotinsky reaction (BZ), which is the standard prototype of nonlinear chemistry. Besides, we used the Python V2.7 programming software and the Jupyter platform for the computational reproduction of the BZ reaction. The STEM methodology could help the development of new competences for students; that is, it will provide them with tools to solve complex current problems that require the interdisciplinarity.
\end{abstract}

Keywords: STEM methodology; teaching; multidiscipline; Belousov-Zhabotinsky reaction (BZ); nonlinear chemistry; python V2.7; reactiondiffusion system.

Se propone una metodología integradora para la enseñanza de la ciencia a través de la metodología STEM (Ciencia-Tecnología-IngenieríaMatemáticas), por sus siglas en inglés. La metodología STEM brinda a los estudiantes la oportunidad de combinar el conocimiento de manera interdisciplinaria y colaborativa, lo que favorece el desarrollo del pensamiento creativo y sistémico. Como modelo, se sugiere la reproducción experimental de la reacción Belousov-Zhabotinsky (BZ), que es el prototipo estándar de la química no lineal. Además, se utilizan el lenguaje de programación Python V2.7 y la plataforma Jupyter para la reproducción computacional de la reacción BZ. La metodología STEM podría ayudar al desarrollo de nuevas competencias para los estudiantes; es decir, les proporcionará herramientas para resolver problemas actuales complejos que requieren de la interdisciplinariedad.

Descriptores: $\quad$ Metodologia STEM; enseñanza; multidisciplina; reacción Belousov-Zhabotinsky (BZ); química no lineal; python V2.7; sistemas de reacción-difusión.

PACS: $01.40 . \mathrm{gb} ; 05.45 .-\mathrm{a} ; 47.20 . \mathrm{Ky} ; 03.40 . \mathrm{Gc}$

\section{Introduction}

STEM studies (Science-Technology-Engineering-Mathematics) are of great importance for the economic development of any country [7]. Martini et al., propose that it is necessary to implement the STEM methodology to create a Maker culture in the population, that is, a community that is highly resilient and that seeks solutions to real problems with a holistic approach and through integrative tools. However, to achieve this, teachers must be able to establish Makerspaces
DOI: https://doi.org/10.31349/RevMexFisE.17.178

[27]. On the other hand, Dómeneche proposes project-based learning as a strategy to implement STEM studies and also describes the incorporation of arts and humanities to provide creativity to STEAM studies (Science-TechnologyEngineering-Arts-Mathematics). The implementation of the arts and humanities, seeks that individuals develop the soft - skills, which is related to the ability to relate to other individuals, collaborative work, communication skills, etc [11]. When implementing STEAM studies, it is sought that individuals, in addition to possessing or developing scientific 
skills, also acquire skills related on many occasions to emotional intelligence $[23,4]$. Nowadays, education centers must develop Makerspaces environments that allow students to build knowledge with a systemic, integrative, and interdisciplinary approach, and, at the same time, allow them to develop their soft - skills, to develop knowledge collaboratively $[3,44]$. The STEM methodology proposes the student as a knowledge builder, capable of developing skills from a holistic perspective. On the other hand, it proposes the teacher as a facilitator or creator of learning environments favorable to the student, while using technology as an activator, driver, or stimulator of learning [42,7,32,50]. In STEM studies, multiple disciplines are ranging from biology, chemistry, physics, and mathematics to highly specialized professions, such as chemical engineering, materials science, etc [7]. All of them can be used together to solve real problems. Students must break the barriers between disciplines and address problems with an interdisciplinary approach (see Fig. 1) $[46,10,9]$. Therefore, in the present work, the experimental reproduction of the Belousov-Zhabotinsky reaction using the STEM methodology is proposed. Students will be able to combine tools of mathematical modeling, programming, chemical kinetics, and experimental chemistry to understand the dynamics that underlie the Belousov-Zhabotinsky reaction, in such a way that students construct knowledge productively and integrally.

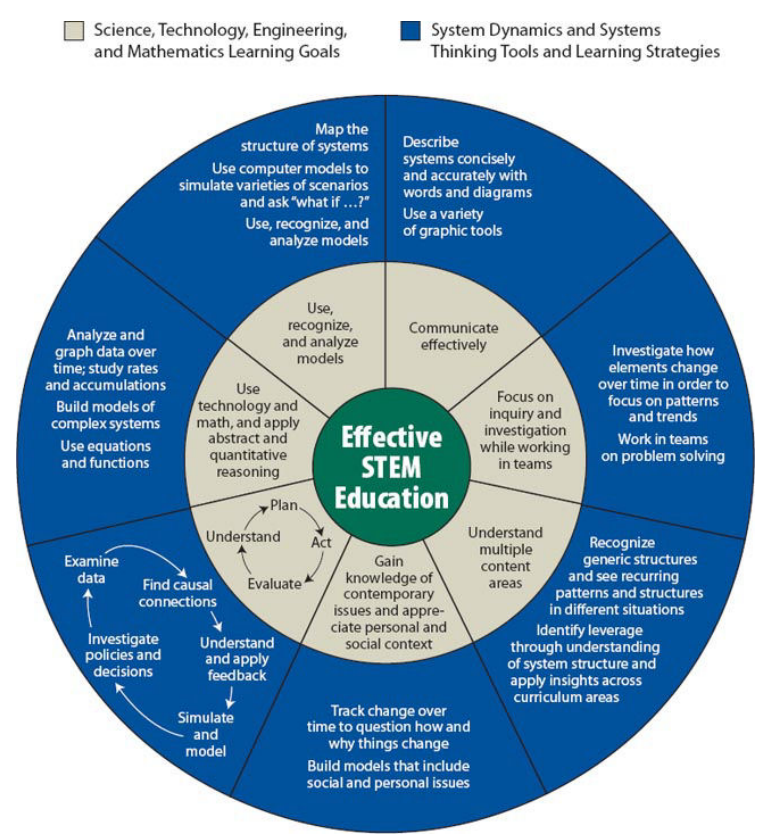

FIGURE 1. STEM approach based on systems dynamics and systems thinking. Source: Creative Learning Exchange (http:// www.clexchange.org/curriculum/standards/stem.asp).

\subsection{The $\mathrm{BZ}$ reaction}

All chemical reactions (or physical changes) in a closed homogeneous system experience a decrease in the free energy of the system. A direct consequence of this principle is that, in such chemical systems, no oscillating changes must be observed around the state of equilibrium since that would imply positive free energy variations, contrary to what is said by thermodynamics $[24,13]$. However, when intervening shortlived intermediary products in the global reaction, the situation becomes more complicated. Normally these intermediate products acquire a stationary concentration or go through a maximum or a minimum, but under certain conditions, the concentrations of the intermediate products oscillate around the expected values in the steady state; that is, their concentrations vary abruptly in the sense of increasing or decreasing their absolute values, a fact that initially baffled the chemists $[17,13]$. This temporary increase was considered a violation of the second law of thermodynamics, and until 1950, many chemists claimed that an oscillating chemical reaction contradicted natural laws [14]. Rudolf Clausius, a German physicist, formulated the fundamental law that occupies us in the following sense: Entropy, the disorder of the Universe, continuously increases through spontaneous reactions [14]. According to this, chemical reactions should evolve continuously to a state of equilibrium. This thermodynamic law did not cover the global aspect, as the experiments of the Russian chemist B.P. Belousov in the year 1950 did show [13].

In the $\mathrm{BZ}$ reaction, it is possible to observe multiple dynamic states, ranging from periodic oscillations in time and space, to episodes of intermittent chaos when it is coupled with other oscillators $[47,17,41,13,55,2]$. When Belousov tried to understand the biochemistry of the Krebs cycle, he proposed the $\mathrm{BZ}$ reaction as an analogous chemical mechanism. He used a solution of bromate and citric acid anions in an acidic medium in the presence of cerium. Then Belousov observed changes in coloration in the solution; the mixture turned yellow when the cerium ions were in their oxidized state, while the solution changed to a transparent tone when the cerium ions were in their reduced state. Subsequently, Zhabotinsky proposed an alternative methodology, changing citric acid to malonic acid and cerium ions for the ferroin indicator. He found besides observing temporal oscillations, spatial oscillations in the form of waves, when the volume of the solution was decreased, and the stirring of the medium was removed [13,41,55]. Years later, Field, Körös and Noyes proposed a general mechanism for the BZ reaction and built the first kinetic model as well as the first numerical simulation of the $\mathrm{BZ}$ reaction $[16,53,15]$. To understand the complex dynamics underlying the BZ reaction, it is necessary to use nonlinear mathematical models that allow us to explain quantitatively or qualitatively the temporal and spatial evolution of this reaction. However, the use of nonlinear mathematical models requires computational simulation tools. A useful tool is the Python programming language [21]. 


\subsection{Python programming language}

Python is a programming language suitable for both scientific computing and teaching, and it is a programming language that can be used with multiple interpreters or development environments. Here are the most commonly used Python distributions:

- Anaconda: www . anaconda.org/anaconda/python

- Enthought Canopy: www . enthought.com

- PyCharm: www.jetbrains.com/pycharm/

Python is a multi-paradigm programming language, which, thanks to its wide variety of packages, can be used for multiple tasks. It has libraries and modules for various applications ranging from numerical calculation (Numpy: WwW.numpy.org/), scientific computing (Scipy: WWW.scipy.org), analysis and data processing (Pandas: WWW . pandas . pydata . org), Machine Learning (scikitlearn: wWW.scikit-learn.org), as well as for specific areas such as chemical engineering (Cantera: WwW . cantera . org). Python is free software (freeware) and exists for all operating systems and can be easily linked with other programming languages such as $\mathrm{C}$ (wWw.cython.org/), Fortran (wWW. fortranwiki.org/fortran/show/Python), Java (http: //www.jython.org/), R (www.rpy2.bitbucket.io/), and specialized software such as Matlab (www.pypi. python.org/pypi/pymatbridge). Next, we write the code in Python of the program that simply shows the word "Hello World"; in Python, it is written as simple as:

In [ ]: print ('Hello_World')

As well as the graph of mathematical functions:

In [ ]: import numpy as $\mathrm{np}$ import matplotlib.pyplot as plt $\mathrm{X}=[]$

$\mathrm{Y}=[]$

for $\mathrm{x}$ in $\mathrm{np}$. arange $(\theta, 100,10)$ :

$\mathrm{y}=\mathrm{x} * * 2$

$\mathrm{X}$. append $(\mathrm{X})$

$Y$. append $(y)$

plt.plot (X,Y, 'rp')

or, written in another form:

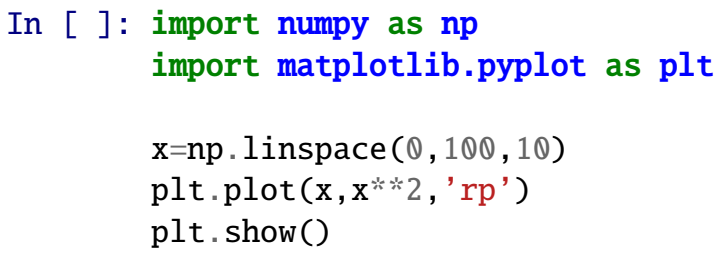

Python is a versatile programming language. Therefore, in this paper, we propose to this programming language as a fundamental tool for learning STEM.

\section{Methodology}

The methodology is divided into three sections, the first of which consists of the mathematical description of the BZ reaction. A system of nonlinear differential equations is proposed to describe the dynamics underlying the BZ reaction. The second section describes the computational methodology for the numerical solution of the model proposed in Sec. 2.1. Finally, the third section consists the experimental reproduction of the $\mathrm{BZ}$ reaction. The student, with the help of the teacher, must correlate the results obtained from the proposed methodologies, with the aim of the student building knowledge with a systemic approach.

\subsection{Theoretical Methodology}

The kinetic scheme originally proposed by Field, Körös, and Noyes (FKN) is the most common chemical mechanism to describe the dynamics of the BZ reaction [13]. Nevertheless, it is not the only one, there are different chemical models that try to explain the dynamics of the reaction $\mathrm{BZ}$ considered that intermediate chemical species, such as $\mathrm{MA}^{*}$ or $\mathrm{Br}^{*}$ would play an important role in the feedback mechanism of the reaction, such is the case of the Radicalator [22,18,29]. However, the FKN model has proved so robust that it is possible to reduce it to a much simpler model called Oregonator $[41,13]$. To obtain all these mathematical models, it is possible to use the standard techniques of chemical kinetics [28].

The overall chemical equation of the $\mathrm{BZ}$ reaction is as follows [19]:

$$
\begin{aligned}
3 \mathrm{BrO}_{3}^{-} & +5 \mathrm{CH}_{2}(\mathrm{COOH})_{2}+3 \mathrm{H}^{+} \rightarrow 3 \mathrm{BrCH}(\mathrm{COOH})_{2} \\
& +4 \mathrm{CO}_{2}+5 \mathrm{H}_{2} \mathrm{O}+2 \mathrm{HCOOH}
\end{aligned}
$$

After several years of research, the BZ reaction mechanism could be elucidated, encountering a mechanism consisting of 27 chemical species and a total of 80 chemical reactions [22,19].

The Oregonator can be obtained from a very simple reaction mechanism composed essentially of five irreversible steps [41]. By the following change of variable, $A=\mathrm{BrO}_{3}^{-}$; $\mathrm{B}=$ organic species such as malonic acid and bromo malonic acid; $P=\mathrm{HOBr} ; X=\mathrm{HBrO}_{2} ; Y=\mathrm{Br}^{-} ; Z=$ the oxidized form of the catalyst; $f$ is a stoichiometric coefficient [41]:

$$
\begin{aligned}
A+Y & \rightarrow X+P \\
X+Y & \rightarrow 2 P \\
A+X & \rightarrow 2 X+2 Z \\
2 X & \rightarrow A+P \\
B+Z & \rightarrow \frac{1}{2} f Y .
\end{aligned}
$$


Whose laws of reaction rate are [41]:

$$
\begin{aligned}
& v_{2}=k_{2}[A][Y] \\
& v_{3}=k_{3}[X][Y] \\
& v_{4}=k_{4}[A][X] \\
& v_{5}=k_{5}[X]^{2} \\
& v_{6}=k_{6}[B][Z] .
\end{aligned}
$$

To construct the Oregonator model, it is necessary to assume that the concentrations of $A$ and $B$ remain constant (they are associated with the initial concentrations of the precursors). Subsequently, it has to apply the standard techniques of chemical kinetics to obtain the dynamic model considering $X, Y$, and $Z$ as dynamic variables, assuming that chemical reactions are elementary, that is, stoichiometric coefficients coincide with the power of dynamic variables [41,28]. The speed laws for Eqs. (2) - (6) are shown in Eqs. (7) - (11). The equations of speed for the variables $X, Y$, and $Z$ are:

$$
\begin{aligned}
& \frac{d[X]}{d \tau}=k_{2}[A][Y]-k_{3}[X][Y]+k_{4}[A][X]-2 k_{5}[X]^{2}, \\
& \frac{d[Y]}{d \tau}=-k_{2}[A][Y]-k_{3}[X][Y]+1 / 2 f k_{6}[B][Z], \\
& \frac{d[Z]}{d \tau}=2 k_{4}[A][X]-k_{6}[B][Z] .
\end{aligned}
$$

Where the $k_{i}$ corresponds to the reaction rate constants and $\tau$ is the time. The analysis is simplified by converting these equations into a dimensionless form [41]. Using the following variable changes:

$$
\begin{aligned}
& x \equiv \frac{2 k_{5}[X]}{k_{4}[A]}, \quad y \equiv \frac{k_{3}[Y]}{k_{4}[A]}, \\
& z \equiv \frac{k_{c} k_{5}[B][Z]}{\left(k_{4}[A]\right)^{2}}, \quad t \equiv k_{c}[B] \tau .
\end{aligned}
$$

Multiplying both sides of Eq. (12) by $\left(2 k_{5} / k_{4}[A]\right)$

$$
\begin{aligned}
\frac{2 k_{5}}{k_{4}} \frac{d x}{d \tau} & =\left(k_{2}[A][Y]-k_{3}[X][Y]\right. \\
& \left.+k_{4}[A][X]-2 k_{5}[X]^{2}\right) \frac{2 k_{5}}{k_{4}[A]}
\end{aligned}
$$

distributing terms,

$$
\begin{aligned}
= & \frac{2 k_{2} k_{5}}{k_{4}}[A][Y]-\frac{2 k_{3} k_{5}}{k_{4}[A]}[X][Y] \\
& +\frac{2 k_{4} k_{5}}{k_{4}[A]}[A][X]-\frac{2 k_{5}^{2}}{k_{4}[A]}[X]^{2}
\end{aligned}
$$

factorizing $k_{4}[A]$,

$$
\begin{gathered}
=k_{4}[A] \frac{2 k_{2} k_{5}}{k_{4}}[A][Y]-k_{4}[A] \frac{2 k_{3} k_{5}}{k_{4}[A]}[X][Y] \\
+k_{4}[A] \frac{2 k_{4} k_{5}}{k_{4}[A]}[A][X]-k_{4}[A] \frac{2 k_{5}^{2}}{k_{4}[A]}[X]^{2}
\end{gathered}
$$

Realizing the change of variable for $x, y$, and $t$,

$$
\frac{k_{6}[B]}{k_{4}[A]} \frac{d x}{d t}=q y-x y+x-x^{2} .
$$

It is renamed:

$$
\frac{k_{6}[B]}{k_{4}[A]} \equiv \varepsilon .
$$

By performing the same procedure for the variables $y$ and $z$ the following system of nonlinear differential equations is obtained [41]:

$$
\begin{aligned}
& \frac{d x}{d t}=\frac{q y-x y+x(1-x)}{\varepsilon} \\
& \frac{d y}{d t}=\frac{-q y-x y+f z}{\varepsilon^{\prime}} \\
& \frac{d z}{d t}=x-z,
\end{aligned}
$$

where $\varepsilon^{\prime} \equiv\left(2 k_{5} k_{6}[B] / k_{3} k_{4}[A]\right)$ and $q \equiv\left(2 k_{5} k_{2} / k_{3} k_{4}\right)$. The typical values of the parameters are: $\varepsilon \approx 10^{-2}, \varepsilon^{\prime} \approx 10^{-5}$, $q \approx 10^{-4}$, and $t$ it is dimensionless time. As the parameter $\varepsilon^{\prime} \approx 10^{-5}$, it is possible to consider the approximation of the steady-state of the variable $y$, therefore, $y \equiv(f z / q+x)$ then the Eqs. (15) - (17) are reduced to [41]:

$$
\begin{aligned}
\varepsilon \frac{d x}{d t} & =x(1-x)+f \frac{q-x}{q+x} z \\
\frac{d z}{d t} & =x-z
\end{aligned}
$$

In this way, the Oregonator model shows the typical form of a chemical feedback system, that is, the variable $x$ functions as an activator, while the variable $z$ has the role of the inhibitor [41]. This model is so robust that it describes the phenomenology of the BZ reaction. The Eqs. (18) - (19) correspond to a system of dimensionless nonlinear differential equations. If to the Eqs. (20) - (21) terms associated with diffusion are added, the system becomes:

$$
\begin{aligned}
\varepsilon \frac{d x}{d t} & =x(1-x)+f \frac{q-x}{q+x} z+D_{x} \nabla^{2} x \\
\frac{d z}{d t} & =x-z+D_{z} \nabla^{2} z,
\end{aligned}
$$

where $D_{x}$ and $D_{y}$ are the dimensionless diffusion coefficients, and $\nabla^{2}$ is the Laplacian operator (see Appendix A). To solve the system of Eqs. (18) - (19) and the system of Eqs. (20) - (21), it is necessary to implement numerical methods and computational simulation tools.

\subsection{Computational Methodology}

For the numerical solution of the Eqs. (18) - (19), the Euler numerical method was used with the following values of the parameters: $\varepsilon \equiv 0.03, q \equiv 0.015$ and $f \equiv 1.0$, with a step of time $d t \equiv 0.0001$. The numerical values of the parameters were selected according to the linear stability criteria for the Eqs. (18) - (19). With these parameter values, the system shows periodic oscillations, which correspond to a limit cycle [34]. The code in Python would be: 
In [ ]: \#Import modules import numpy as $\mathrm{np}$ \#Module for arrays import matplotlib.pyplot as plt \#Module for graphics

\#Model parameters

$\mathrm{f}=1.0$

$\mathrm{q}=0.015$

$\mathrm{e}=0.03$

\#Numerical method parameters

tQ $=0.0$ \#Initial time

$\mathrm{t} f=100.0$ \#Final time

$\mathrm{dt}=0.0001$ \#Time step

\#Initial Condition

$\mathrm{X} Q=0.5$

$\mathrm{ZQ}=0.5$

\#Functions

def $\operatorname{fun} 1(x, z)$ :

return $\left(x^{*}(1-x)\right.$

$+f * z *((q-x) /(q+x))) / e$

$\operatorname{def} \operatorname{fun} 2(x, z)$ :

return $x-z$

\#Lists for data

$\mathrm{X}=[]$ \#List for $\mathrm{X}$ variable

$\mathrm{Z}=$ [] \#List for $z$ varibale

$\mathrm{T}=[]$ \#List for time values

\#Assign initial condition

$\mathrm{X}=\mathrm{X} \theta$

$\mathrm{Z}=\mathrm{ZQ}$

\#Numerical method

for $t$ in $n p$.arange $(t \mathbb{Q}, t f, d t)$ :

$\mathrm{t}=\mathrm{t}+\mathrm{dt}$

\#Euler rule for $x$ variable

$\mathrm{x}=\mathrm{fun} 1(\mathrm{x}, \mathrm{z}) * \mathrm{dt}+\mathrm{x}$

\# Euler rule for $z$ variable

$\mathrm{z}=\mathrm{fun} 2(\mathrm{x}, \mathrm{z}) * \mathrm{dt}+\mathrm{z}$

$\mathrm{X}$. append $(\mathrm{x})$ \#List for $\mathrm{x}$ variable

$Z$. append( $z$ ) \#List for $z$ variable

$\mathrm{T}$.append $(\mathrm{t})$ \#List for $t$ variable

\#Plot construction

$f$, axes $=$ plt. $\operatorname{subplots}(1,2$,

figsize $=(14,6))$

axes [0].plot (T, X, 'b. ', label='x')

$\operatorname{axes}[0] \cdot \operatorname{plot}\left(\mathrm{T}, \mathrm{Z}, \mathrm{\prime}^{\prime} \mathrm{k}\right.$ ', label=' $\mathrm{Z}$ ')

axes[0].set_title('Oscillations',

$\operatorname{size}=16$ )

axes[0].set_xlabel('t [adimensional]',

size $=14$ )

axes[0].set_ylabel('x,z [adimensional]',

size=14)

axes $[\theta]$. legend (loc=' best')

axes[1].plot (X,Z,'g*', label='Phase Space')

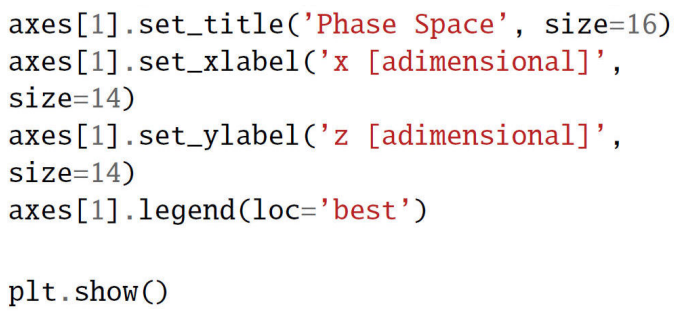

For the numerical solution of the Eqs. (20) - (21), the numerical method of discretization by finite differences was used, under the condition of Neumann contour. The following values of the parameters were used: $\varepsilon \equiv 0.03, q 0.015$, $f \equiv 2.0$, with a time step $d t \equiv 0.001$, a spatial step $d h \equiv 0.5$, $D_{x} \equiv 2.0$ and $D_{z} \equiv 0.0$. The code used to numerically integrate the Eqs. (20) - (21) is described below.

\section{In [ ]: from Larray import* \\ \#Reaction-Diffusion module}

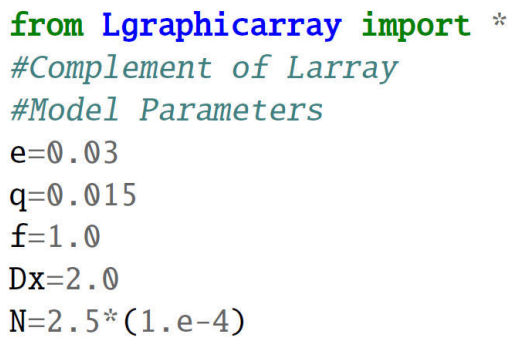




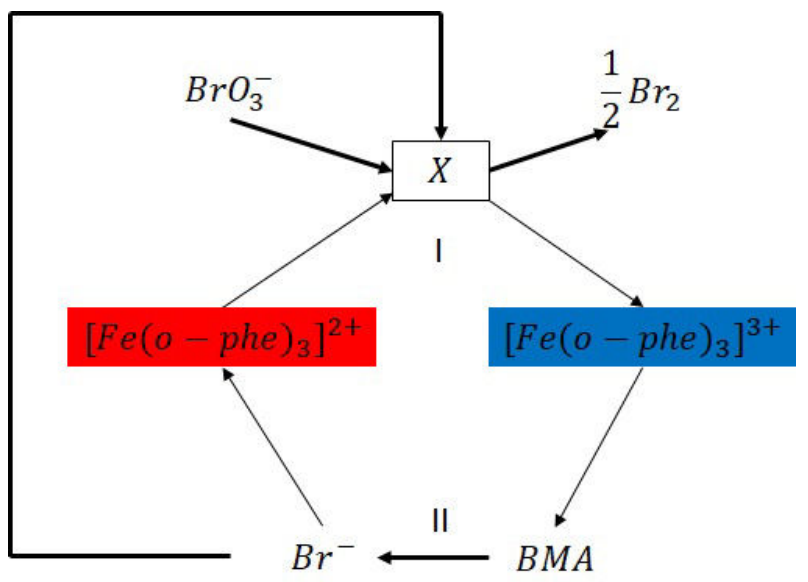

Figure 3. The general feedback mechanism of the BZ reaction. Modified from [55].

Essentially, the BZ reaction mechanism consists of two stages, the first one consists in the oxidation of the ferroin indicator by the bromate anion (obtained from the potassium bromate precursor), which gives the solution the blue color. On the other hand, the second stage consists of the reduction of the ferroin indicator in the presence of malonic acid, observing a red coloration in the solution. During the progress of the reaction, the bromate anion allows the production of $\mathrm{HBrO}_{2}$, which facilitates the oxidation process of the indicator. On the other hand, organic compounds derived from bromine (BMA) are formed, which favor the production of bromide ions $\left(\mathrm{Br}^{-}\right)$and reduce the ferroin indicator; however, bromide ions act as strong inhibitors of the feedback mechanism. An outline of the general feedback mechanism can be seen in the Fig. 3 [55].

This process corresponds to a state of self-organization of the system, which results in the emergence of collective properties (oscillations) [48]. The formation of dissipative structures corresponds in the same way to a state of selforganization of the system or self-organized criticality. In this state, the system by itself tends to self-organize without the need for an external disturbance (see Fig. 3) $[8,13,25]$. The processes of self-organization are present in a great variety of biological systems. The oscillations of concentrations of chemical species are observed in processes such as the hormonal regulation of the thyroid [5], the insulin-leptin axis [36], which is essential for the blood glucose regulation [20], hormone regulation in the menstrual cycle in mammals [49], etc. Disturbances in such oscillatory physiological processes lead to the emergence of pathologies [35]. On the other hand, dissipative structures (spatiotemporal patterns) appear in biological phenomena, such as the formation of patterns in the skin of mammals and fish [30], formation of bacterial colonies [6], crop growth [54] etc.

The numerical solution of the Eqs. (18)-(19) reproduces the oscillatory behavior of the BZ reaction (see Fig. 4a). In Fig. (4b), it is clear that these oscillations emerge due to the existence of a limit cycle in the phase space of variables [16]. In the limit cycle orbits or system solutions of Eqs. (18)-(19)
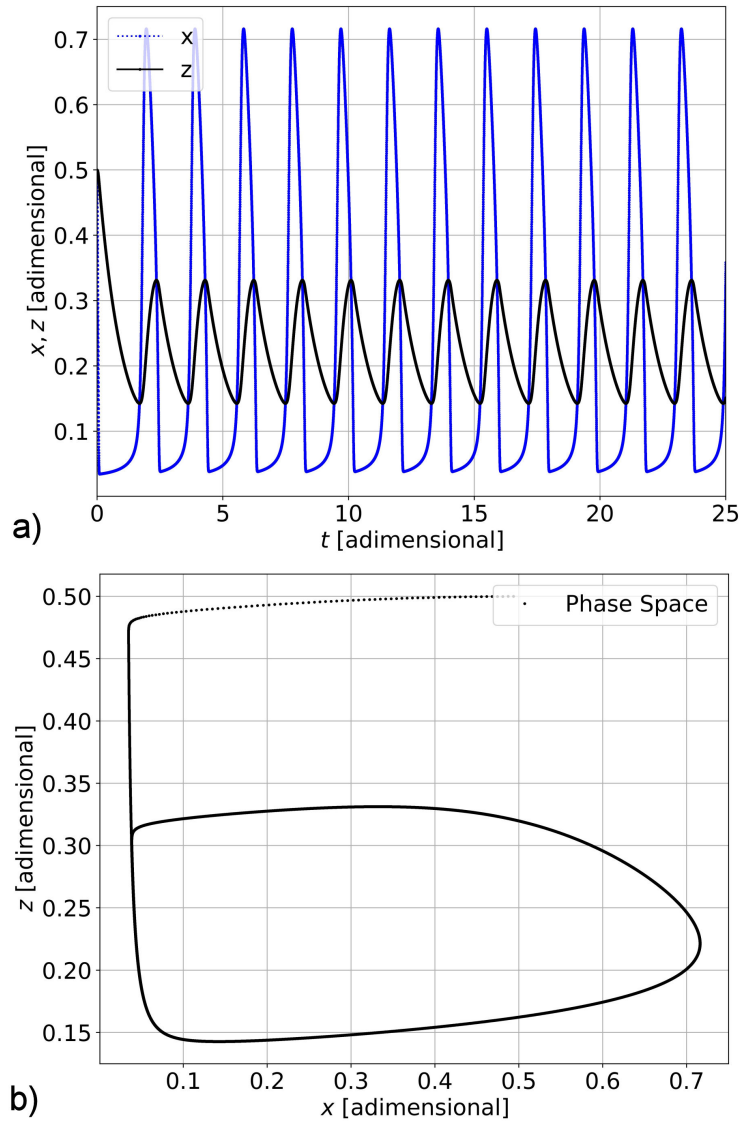

FIgURE 4. Graphs obtained from the numerical solution of the Eqs. (18)-(19). a) Oscillations of the variables $x$ y $z$ due to the change in the oxidation state of the ferroin indicator. b) Phase space of the variables $x$ and $z$, the presence of a limit cycle is observed [48].

revolve around a fixed point, equilibrium point, or steadystate. The Eqs. (18)-(19) only qualitatively explain the dynamics of the BZ reaction, that is, they explain only the existence of oscillations. However, it is important to note that the Eqs. (18)-(19) can be modified to explain in more detail the dynamics of the BZ reaction $[26,1]$.

In Figs. (5b)-(5c) appears the numerical solution of the Eqs. (20)-(21). The formation of spatio-temporal patterns which corresponds to what was observed in the experimental reproduction of the $\mathrm{BZ}$ reaction (see Fig. 5a). However, it is necessary to mention that Eqs. (20)-(21) only qualitatively explain the spatial-temporal dynamics of the $\mathrm{BZ}$ reaction, and the spatio-temporal patterns that emerge from the dynamics of the $\mathrm{BZ}$ reaction [51].

During the development of the methodology, the student will be able to construct knowledge systemically and integrally, addressing a real problem and implementing tools of Chemistry, Physics, Biology, Mathematics, and Computing. This methodology will allow students to pose new questions and hypotheses It will give them the possibility to explore and build knowledge in a creative way. Besides, the teacher could propose new questions to motivate the creative learning of 


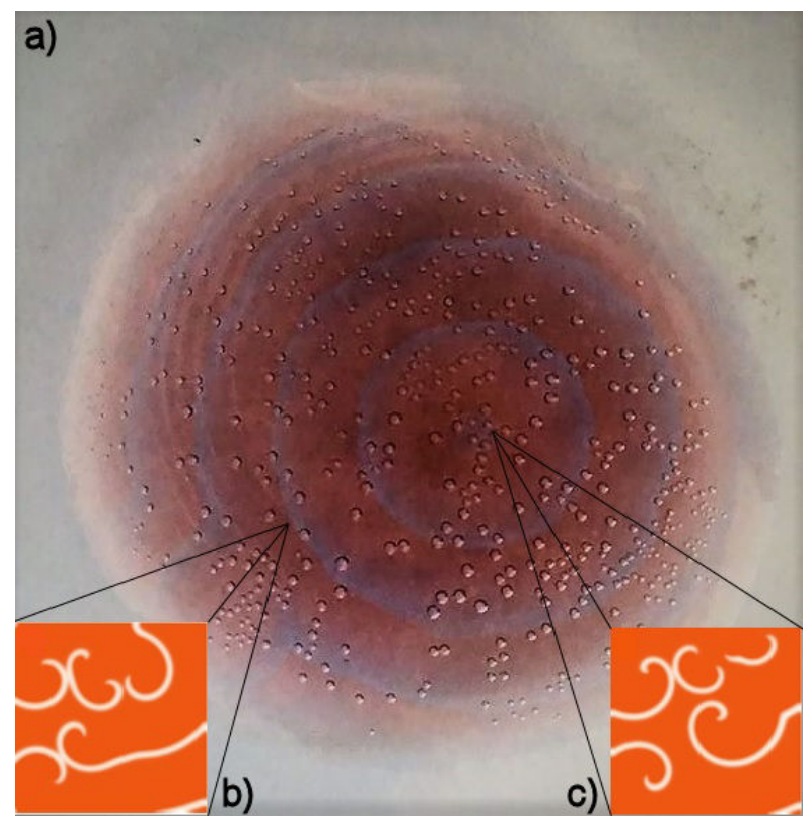

FIGURE 5. a) Formation of chemical waves in the BZ reaction in the absence of agitation. b), c). Numerical solution of the Eqs. (20) (21). Showing the formation of chemical waves.

the students. Here we propose some topics that may be of interest during the development of the methodology.

- What could happen if the concentration of sulfuric acid in the medium is changed?

- How could the action of $\mathrm{pH}$ modify the dynamics of Eqs. (18)-(19)?

- The concentration of sulfuric acid could influence the formation of spatio-temporal patterns?

- If you change the concentration of potassium bromate and potassium bromide, would you modify the frequency of the oscillations?

- What happens if the agitation in the beaker is removed during the chemical reaction?

- Is it possible to observe the formation of chemical waves in 3D during the experimental procedure? If this is possible, what variable has to be modified to observe chemical waves?

- It can be considered that the beaker is a "Batch" type reactor. How could a continuous system (CSTR) be implemented? How would the system of Eqs. (18)(19)? What parameters would have to be added to the model? How would you modify the dynamics of the model?

$$
\begin{aligned}
\frac{d x}{d t} & =F(x, z, q, \epsilon, f, \text { flows, volume }) \\
\frac{d z}{d t} & =G(x, z, \text { flows }, \text { volume })
\end{aligned}
$$

- The system of Eqs. (18)-(19) arises from a material balance considering the system as a Batch reactor. Is it possible to build an energy balance for the system to study the system temperature $(T)$ ? How would the system of Eqs. (18)-(19)?

$$
\begin{aligned}
\frac{d x}{d t} & =F(x, z, T, q, \epsilon, f, \text { flows, volume }) \\
\frac{d z}{d t} & =G(x, z, T, \text { flows, volume }) \\
\frac{d T}{d t} & =H(x, z, T, q, \epsilon, f, \text { flows, volume }) .
\end{aligned}
$$

- How do the dynamics of the Eqs. (18)-(19), if the variables and parameters of the initial conditions model are modified? Modify the Python program of the Euler numeric method.

- How is the dynamic of the Eqs. (20)-(21), if the value of the dimensionless diffusion coefficient is changed? If this parameter is modified, are we still observing spatio-temporal patterns?

- Can the codes built in Python be used to solve numerically other kinetic models?

- The teacher can use the methodology proposed in this paper to propose the theoretical, computational, and experimental study of other oscillating reactions, such as:

$$
\begin{aligned}
& \text { - Alternator [37]. } \\
& \text { - Explodator [31]. } \\
& \text { - Brusselator [33]. } \\
& \text { - Berlinator [31]. } \\
& \text { - Braylator [43]. }
\end{aligned}
$$

- Why did Belousov and Zhabotisnky implement the BZ reaction as a strategy to study glycolysis and the Krebs cycle, respectively? What substance in the $\mathrm{BZ}$ reaction is used as a chemical species analogous to citric acid?

- Students at more advanced levels can perform the linear stability analysis of eqs. (18) - (21) to study how system dynamics change when one or more parameters of the model are modified (see Fig. 6) [48]. Students will find that the Oregonator model presents a HopfAndronov bifurcation [48], and when the Eqs. (18)(19) are modified, the presence of bistability, hysteresis, and chaos is observed $[40,38,52,12,45]$.

- The teacher can create interactive scripts using the ipywidgets library (www.ipywidgets.readthedocs. io/en/latest/index.html). Below is an example of an interactive script for solving the Eqs. (18)-(19).

- A function that contains the Euler numerical method for the solution of the Eqs. (18)-(19). Once the script is built, it will be saved with the name bz.py: 


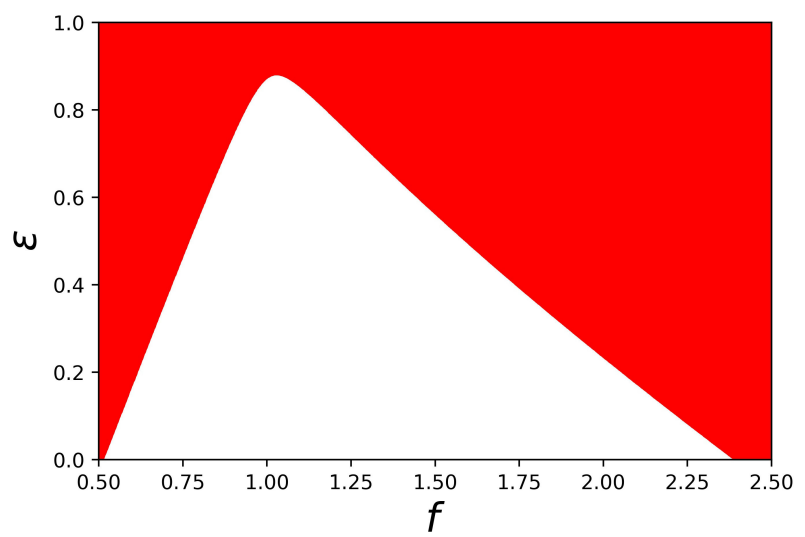

FIGURE 6. Parameter space obtained from the linear stability analysis [48] $(q=0.0008)$. White and red regions show limit cycles and damped oscillations, respectively.

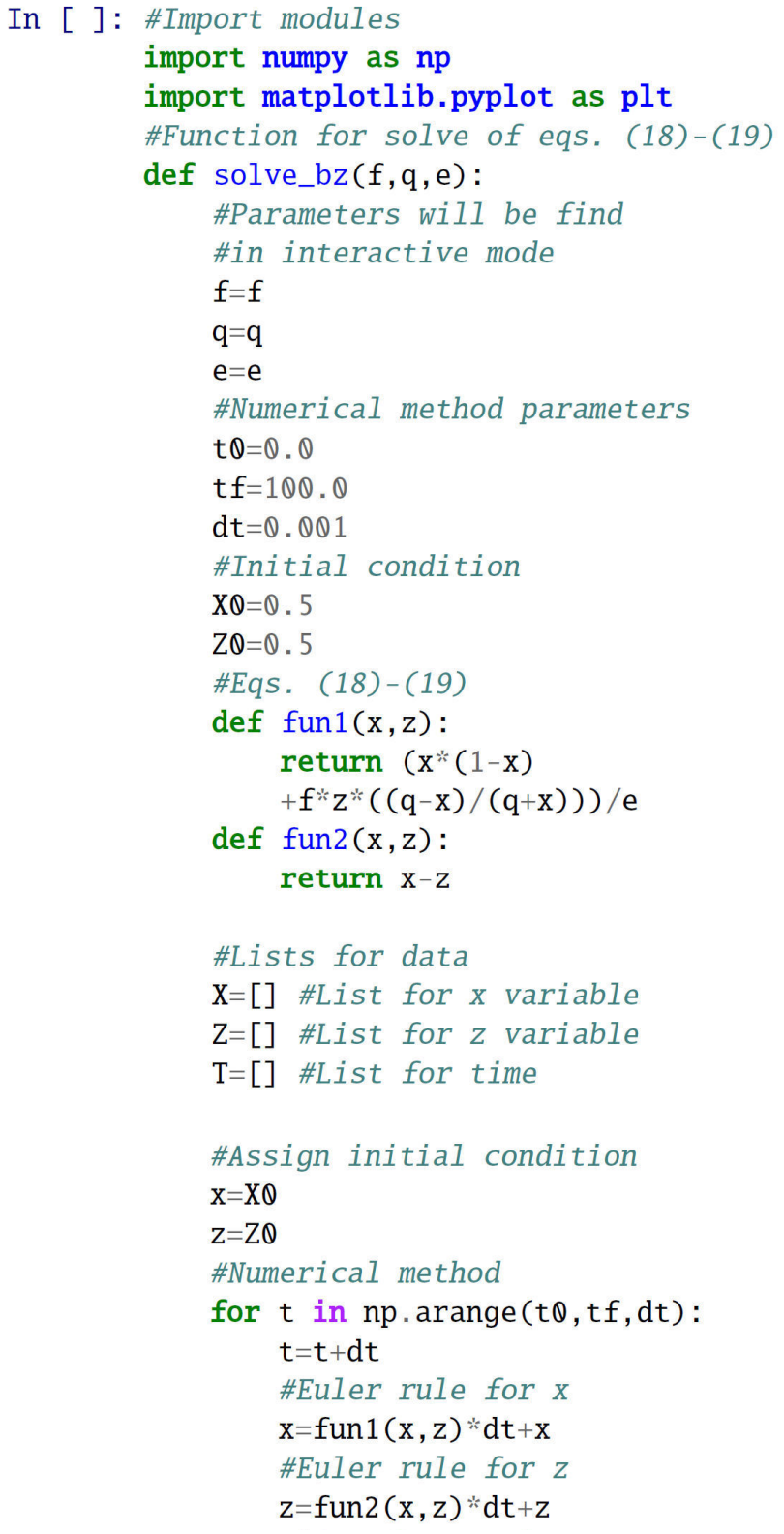

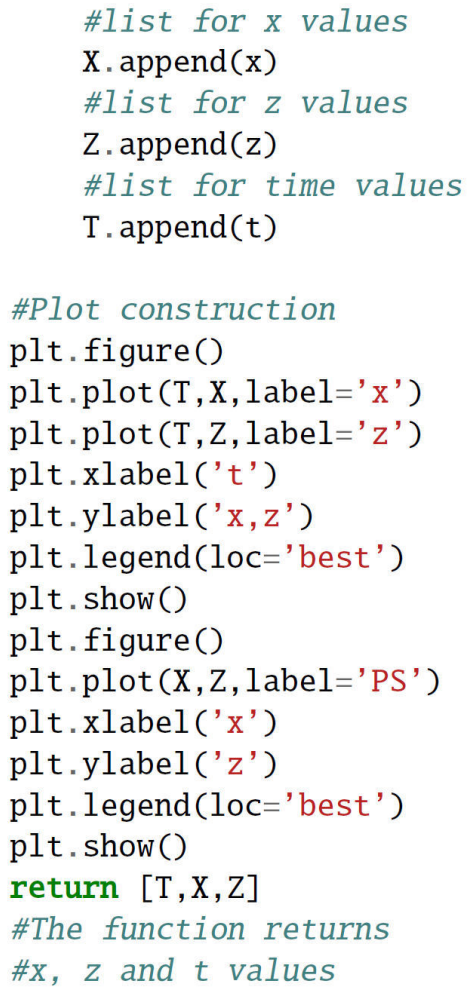

- Later, a script is created that has the instructions of the interactive mode, which must be saved in the same folder as the bz.py script:

In [ ]: \#Import modules \%matplotlib inline from ipywidgets import interactive \#Interactive mode module from bz import solve_bz \#Calling the function above int=interactive(solve_bz, $\mathbf{f}=(\theta, 5 . \theta), \mathbf{q}=(\theta .015,1.0)$, $\mathrm{e}=(\theta .03,1.0))$

int

Figures (7)-(8) shows the numerical solution of the Eqs. (18)-(19) for different values of the parameters of the model using the interactive mode. In interactive mode, students can easily modify parameter values and observe the change in system dynamics. The teacher can use the previous script to study the dynamics of other kinetic models $[37,31,33,43]$.

- The proposed methodology can be used to explain to students the relationship that exists between different areas of knowledge. Students must understand that each of the different areas of science are interrelated. 

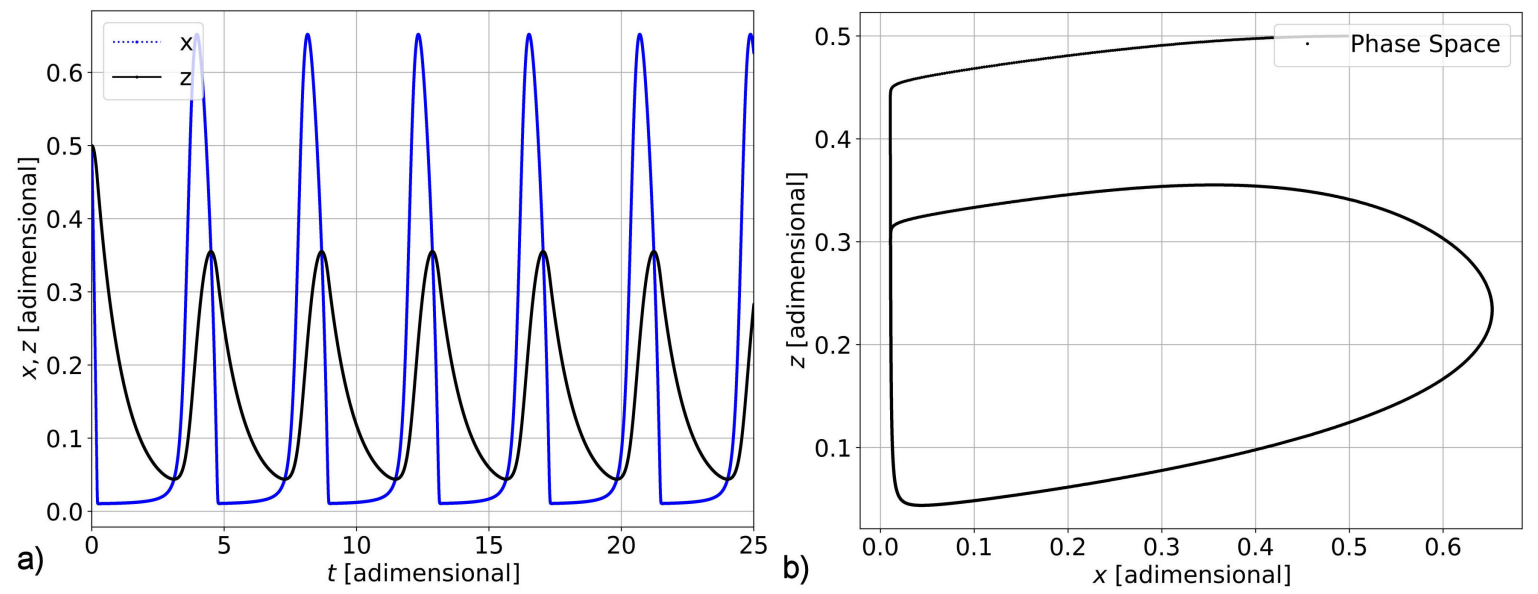

FIGURE 7. Numerical solution of the eqs. (18) - (19) with $f=1.0, q=0.015$ and $\epsilon=0.11$. a) Periodic oscillations of the variables $x$ and $z$. b) Phase space, showing a limit cycle [48].
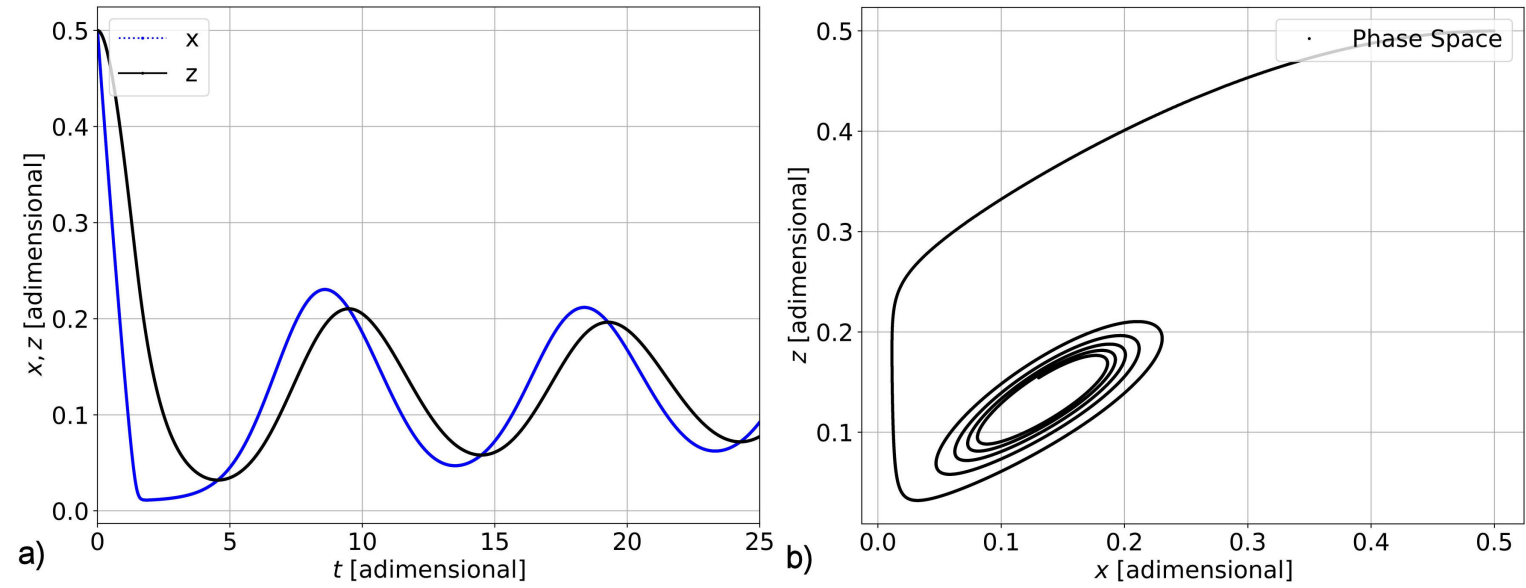

Figure 8. Numerical solution of the Eqs. (18)-(19) with $f=1.0, q=0.015$ and $\epsilon=0.61$. a) Damped oscillations of the variables $x$ and $z$. b) Phase space. The dynamics of the system changes as a result of the fixed point [48].

\section{Conclusion}

The $\mathrm{BZ}$ reaction is one of the ideal prototypes for the application of STEM studies because it allows the implementation and combination of different areas of science (Biology, Chemistry, Physics, Mathematics, and Computing). During the development of the methodology, students will systemically build knowledge understand that the different areas of science are interrelated and that solving or proposing solutions to a real problem requires an interdisciplinary approach. It is of vital importance that the teaching of science allows students to explore and learn actively, eliminating the traditional forms of teaching, in which students only acquire knowledge passively.

\section{Appendix}

\section{A. Diffusion terms and numerical expressions of them}

These systems can be constructed from a generalized conservation law in which, if a differential volume $V$ surrounded by a closed surface $S$ is filled with a substance whose concentration or density is $x$, then the rate of change of all substance in $V$ will be given by a net flux into or from the volume $V$ across the surface $S$ and by possible sources or sinks due to reactions or another physical, chemical or biological processes into the volume $V$. In this way

$$
\frac{d X}{d t}=\frac{d}{d t} \int_{V} x d v=-\oint_{S} \vec{J} \cdot d \vec{s}+\int_{V} f(x, \ldots) d v .
$$

In last expression, $f(x, \ldots)$ represents sources, sinks, reactions (as in this case), dead or born, etc. in the system.

On the other hand, inert matter generally satisfies the empirical Fick's law $\vec{J}(x)=-D \nabla x$, where $D$ is the diffusion coefficient [39]. So that replacing $\vec{J}(x)$ and applying the divergence theorem in Eq. (A.1):

$$
\int_{V} \frac{d x}{d t} d v=\int_{V} D \nabla^{2} x d v+\int_{V} f(x, \ldots) d v,
$$

which is valid for any arbitrary volume $V$, so that, finally

$$
\frac{d x}{d t}=D \nabla^{2} x+f(x, \ldots),
$$


is the Reaction-Diffusion Equation, and the function $f$, in this case, depends on $x, z$, and some set of parameters such as reaction velocities or reaction constants.

A numerical expression is necessary for the Laplacian operator $\nabla^{2}$, and it can be obtained from Taylor's series of $u(x, y)$ around $x_{0}$ with small $h$ as

$$
\begin{aligned}
u\left(x_{0}+h, y\right) & =u\left(x_{0}, y\right)+\left.h \frac{\partial u}{\partial x}\right|_{x_{0}} \\
& +\left.\frac{h^{2}}{2 !} \frac{\partial^{2} u}{\partial x^{2}}\right|_{x_{0}}+\ldots
\end{aligned}
$$

and

$$
\begin{aligned}
u\left(x_{0}-h, y\right) & =u\left(x_{0}, y\right)-\left.h \frac{\partial u}{\partial x}\right|_{x_{0}} \\
& +\left.\frac{h^{2}}{2 !} \frac{\partial^{2} u}{\partial x^{2}}\right|_{x_{0}}-\ldots
\end{aligned}
$$

The sum of Eqs. (A.4) and (A.5) gives

$$
\begin{aligned}
u\left(x_{0}+h, y\right) & +u\left(x_{0}-h, y\right)=2 u\left(x_{0}, y\right) \\
& +\left.h^{2} \frac{\partial^{2} u}{\partial x^{2}}\right|_{x_{0}}+O\left(h^{4}\right),
\end{aligned}
$$

which, dividing by $h^{2}$ gives an expression for the second partial derivative of $u(x, y)$ at $x_{0}$, for any $y$ :

$$
\left.\frac{\partial^{2} u}{\partial x^{2}}\right|_{x_{0}}=\frac{u\left(x_{0}+h\right)+u\left(x_{0}-h\right)-2 u\left(x_{0}\right)}{h^{2}}+O\left(h^{2}\right),
$$

so, if we represent $u$ using a mesh such that $u_{i j}$ represents $u(x, y)$ in a surface element of size $h^{2}$, then the discrete version of the second derivative will be

$$
\frac{\partial^{2} u_{i j}}{\partial x^{2}}=\frac{u_{i, j+1}+u_{i, j-1}-2 u_{i j}}{h^{2}},
$$

with an error of $O\left(h^{2}\right)$ order, and the Laplacian operator is

$$
\nabla^{2} u_{i j}=\frac{u_{i, j+1}+u_{i, j-1}+u_{i+1, j}+u_{i-1, j}-4 u_{i j}}{h^{2}} .
$$

\section{Acknowledgments}

We would like to thank the Faculty of Mathematical Physics of the Benemérita Universidad Autónoma de Puebla and the Physics-Chemistry group for the Degree in Applied Physics of the Fall 2017 period, since thanks to their effort it was possible to carry out this work. As Dr. José Manuel Nieto Villar, head of the Department of Chemistry-Physics of the University of Havana in Cuba, would say: "Students must know that they are the perpetuum mobile that transcends any thermodynamic theory". We also thank the Faculty of Chemical Sciences of the Benemérita Universidad Autónoma de Puebla and mainly the Biophysical Chemistry Laboratory and the M.S. Ana María Cervantes Tavera for providing the facilities (chemical reagents, laboratory equipment, and methodology) for the experimental realization of the $\mathrm{BZ}$ reaction. We thank the Python Programming Club of the Chemical Engineering Faculty of the Benemérita Universidad Autónoma de Puebla for their support during the implementation of Python in the present work. A special thanks to the Faculty of Biological Sciences of the Benemérita Universidad Autónoma de Puebla, for being a catalyst agent of talents.
1. T. Amemiya et al. "Spiral wave formation in three-dimensional excitable media". Phys. Rev. Lett. 77.15 (1996) 3244.

2. J. A. Arzola et al. Sincronización en sistemas quimicos. In: Revista Mexicana de Ingeniería Quimica 16 (2017) 883-898.

3. N. Awad and M. Barak, Pre-service Science Teachers Learn a Science, Technology, Engineering and Mathematics (STEM)Oriented Program: The Case of Sound, Waves and Communication Systems. Eurasia Journal of Mathematics, Science and Technology Education 14.4 (2018) 1431-1451. https: //doi. org/10.29333/ejmste/83680

4. R. Bancino and C. Zevalkink, Soft Skills: The New Curriculum for Hard-Core Technical Professionals. Techniques: Connecting Education and Careers (J1) 82.5 (2007) 20-22.

5. J. Berberich et al., Mathematical Modeling of the PituitaryThyroid Feedback Loop: Role of a TSH-T3-Shunt and Sensitivity Analysis". In: Front. Endocrin. 9 (2018) 91. https: //doi.org/10.3389/fendo.2018.00091

6. E. O. Budrene and H. C. Berg, Dynamics of formation of symmetrical patterns by chemotactic bacteria. Nature 376.6535 (1995) 49. https://doi .org/10.1038/376049a0
7. D. Elia Caballero Hernández. "El papel de los estudios STEM en el avance económico y social". Entorno universitario $\mathbf{1 6 . 4 2}$ (2015) 10-12.

8. C. Carrillo, Matemáticas: la gramática de la naturaleza. Vol. 2. Siglo XXI, (2012).

9. X. Chen, "Students Who Study Science, Technology, Engineering, and Mathematics (STEM) in Postsecondary Education. Stats in Brief. NCES 2009-161". National Center for Education Statistics (2009).

10. C. J. Craig et al., The influence of parents on undergraduate and graduate students' entering the STEM disciplines and STEM careers. International Journal of Science Education (2018) 123. https://doi.org/10.1080/09500693.2018.1431853

11. J. Doménech-Casal, Aprendizaje Basado en Proyectos en el marco STEM: componentes didáctica para la Competencia Científica. Rev. Edu. Cienti. 21.2 (2018) 29-42. https://doi . org/10.17979/arec.2018.2.2.4524

12. C. Egami, Mechanism for the color transition of the BelousovZhabotinsky reaction catalyzed by cerium ions and ferroin. In: Discrete and Continuous Dynamical Systems-B (2018) 33-42. https://doi.org/10.3934/dcdsb.2018061 
13. I. R. Epstein, J. A. Pojman, and O. Steinbock, Introduction: Self-organization in nonequilibrium chemical systems. Chaos: An Interdis. J. Nonlinear Sci. 16.3 (2006) 037101. https: //doi.org/10.1063/1.2354477

14. I. R. Epstein et al. Oscillating chemical reactions. Scientific American 248 (1983) 112-123.

15. R. J. Field, E. Koros, and R. M Noyes, "Oscillations in chemical systems. II. Thorough analysis of temporal oscillation in the bromate-cerium-malonic acid system". J. Ame. Chem. Soc. 94.25 (1972) 8649-8664. https://doi.org/10.1021/ ja00780a001

16. R. J. Field and R. M. Noyes, Oscillations in chemical systems. IV. Limit cycle behavior in a model of a real chemical reaction. J. Chem. Phys. 60.5 (1974) 1877-1884. https: //doi.org/10.1063/1.1681288

17. R. J. Field and F. W. Schneider, Oscillating chemical reactions and nonlinear dynamics. J. Chem. Educ. 66.3 (1989) 195. https://doi.org/10.1021/ed066p195

18. H.-D. Försterling, S. Muranyi, and Z. Noszticzius, The role of radicals in the Belousov-Zhabotinsky reaction. React. Kinet. Catal. Lett. 42.2 (1990) 217-226. https://doi.org/10. 1007/BF02065357

19. A. Fuentes Vetán and F. Guillermo Mosqueira. "Una reacción oscilante par alumnos del nivel medio superior". Educ. Quim 16.1 (2015) 99-103.

20. D. Gradinaru et al., Insulin-Leptin Axis, Cardiometabolic Risk and Oxidative Stress in Elderly with Metabolic Syndrome. Experimental and Clinical Endocrinology and Diabetes (2018). https://doi.org/10.1055/s-0043-123825

21. J. E. Grayson, Python and Tkinter programming. Vol. 140. (Manning Greenwich, 2000).

22. L. Gyorgyi, T. Turányi, and R. J. Field, Mechanistic details of the oscillatory Belousov-Zhabotinskii reaction. $J$. Phys. Chem. 94.18 (1990) 7162-7170. https://doi.org/ $10.1021 / \mathrm{j} 100381 \mathrm{a} 039$

23. N. HjYunus and C. Norlia Hassan, The importance of emotional intelligence and soft skills in the workplace. In: 2012 International Conference on Innovation Management and Technology Research. 2012. https://doi.org/10.1109/ICIMTR. 2012.6236352

24. D. Kondepudi, Introduction to modern thermodynamics. (Wiley, 2008).

25. D. Kondepudi and I. Prigogine, Modern thermodynamics: from heat engines to dissipative structures. (John Wiley and Sons, 2014).

26. H. Juergen Krug, L. Pohlmann, and L. Kuhnert. "Analysis of the modified complete Oregonator accounting for oxygen sensitivity and photosensitivity of Belousov- Zhabotinskii systems". J. Phys. Chem. 94.12 (1990) 4862-4866.

27. S. Martini and M. Chiarella, Didáctica Maker. Estrategias colaborativas de aprendizaje STEM en Diseño Industrial. In: Proceedings XX Congreso SIGraDi, (Concepción. Chile. 2017), pp. 158-164. https://doi.org/10.5151/ sigradi2017-025

28. R. I. Masel et al. Chem. Kinet. Catalysis. (Wiley-Interscience New York, 2001).
29. S. Muranyi and H.-D. Forsterling, Chloride Induced Oscillations in the Classical Belousov-Zhabotinsky Reaction Explained by the Radicalator-Model. In: Zeitschrift für Naturforschung A 45.2 (1990) 135-144. https://doi.org/10. 1515/zna-1990-0208

30. H. Frederick Nijhout, Pattern formation in the physical and biological sciences. CRC Press, 2018.

31. Z. Noszticzius, H. Farkas, and Z. A. Schelly, Explodator: A new skeleton mechanism for the halate driven chemical oscillators. J. Chem. Phys. 80.12 (1984) 6062-6070. https://doi. org/10.1063/1.446688

32. C. Ntemngwa and S. Oliver, The Implementation of Integrated Science Technology, Engineering and Mathematics (STEM) Instruction using Robotics in the Middle School Science Classroom. In: Inter. J. Educ. Mathe. Scien. Technol. 6.1 (2018) 12-40. https://doi.org/10.46328/ijemst.v6i1.426

33. B. Pena and C. Perez-Garcia, Stability of Turing patterns in the Brusselator model. Phys. Rev. E 64.5 (2001) 056213. https: //doi.org/10.1103/PhysRevE.64.056213

34. V. Petrov et al., Controlling chaos in the Belousov-Zhabotinsky reaction. In: Nature 361.6409 (1993) 240. https://doi .org/ $10.1038 / 361240 \mathrm{a} 0$

35. V. Pilorz, C. Helfrich-Förster, and H. Oster, The role of the circadian clock system in physiology. Pflügers ArchivEuropean J. Physiology (2018) 1-13. https://doi.org/10. 1007/s00424-017-2103-y

36. D. Porte Jr., D. G Baskin, and M. W. Schwartz. "Leptin and insulin action in the central nervous system" Nutrition reviews suppl 60 (2002) S20-S29.

37. G. Rabai, K. Kustin, and I. R. Epstein, A systematically designed $\mathrm{pH}$ oscillator: the hydrogen peroxide-sulfiteferrocyanide reaction in a continuous-flow stirred tank reactor. J. Ame. Chem. Soc. 111.11 (1989) 3870-3874. https: //doi.org/10.1021/ja00193a018

38. P. Richetti et al. "rom quasiperiodicity to chaos in the BelousovZhabotinskii reaction. II. Modeling and theory". J. Chem. Phys. 86.6 (1987) 3339-3356.

39. J. F. Rojas and M. A. Morales, Experimentos numéricos en el aula sobre fenómenos difusivos: difusión anómala en sistemas físicos y biológicos. Rev. Mex. Fis. 56 (2010) 41-50.

40. P. Ruoff and R. M. Noyes, "An amplified Oregonator model simulating alternative excitabilities, transitions in types of oscillations, and temporary bistability in a closed system". $J$. Chem. Phys. 84.3 (1986) 1413-1423.

41. F. Sagues and I. R. Epstein, Nonlinear chemical dynamics. Dalton transactions 7 (2003) 1201-1217. https://doi .org/10. 1039/B210932H

42. R. Sánchez et al. "Nuestros paisajes educativos están cambiando: la enseñanza y aprendizaje STEM". Entorno universitario 16.43 (2015) 2-6.

43. G. Schmitz, The oxidation of iodine to iodate by hydrogen peroxide. In: Phys. Chemistry Chem. Phys. 3.21 (2001) 47414746.https://doi.org/10.1039/B106505]

44. Engineering National Academies of Sciences, Medicine, et al. Indicators for Monitoring Undergraduate STEM Education. (National Academies Press, 2018). 
45. R. Srivastava, M. Dueñas-Díaz, and J. Pérez-Mercader, Feed rate noise modulates autocatalysis and shapes the oscillations of the Belousov-Zhabotinsky reaction in a continuous stirred tank reactor. Reaction Chem. Engineer. 3 (2018) 216-226. https: //doi.org/10.1039/C7RE00196G

46. G. Stoet and D. C. Geary, The gender-equality paradox in science, technology, engineering, and mathematics education. Psychol. Sci. (2018) 136. https://doi.org/10.1177/ 0956797617741719

47. P. Strizhak and M. Menzinger, Nonlinear dynamics of the BZ reaction: A simple experiment that illustrates limit cycles, chaos, bifurcations, and noise. In: J. Chem. Educ. 73.9 (1996) 868. https://doi.org/10.1021/ed073p868

48. S. H. Strogatz, Nonlinear dynamics and chaos: with applications to physics, biology, chemistry, and engineering. (CRC Press, 2018).

49. L. Taylor-Swanson et al., The dynamics of stress and fatigue across menopause: attractors, coupling, and resilience. Menopause 25.4 (2018) 380-390. https://doi.org/10. 1097/GME. 0000000000001025

50. L. Thibaut et al., The influence of teachers' attitudes and school context on instructional practices in integrated STEM educa- tion. Teaching and Teach. Educ. 71 (2018) 190-205. https: //doi.org/10.1016/j.tate.2017.12.014

51. A. Mathison Turing, The chemical basis of morphogenesis. In: Philosophical Transactions of the Royal Society of London. Series B, Biological Sciences 237.641 (1952) 37-72. https: //doi.org/10.1098/rstb.1952.0012

52. J. S. Turner et al. "Alternating periodic and chaotic regimes in a chemical reaction- experiment and theory". Phys. Lett. A 85.1 (1981) 9-12.

53. J. J. Tyson,Scaling and reducing the Field- Koros-Noyes mechanism of the Belousov- Zhabotinskii reaction. In: The Journal of Physical Chemistry 86.15 (1982) 3006-3012. https: //doi.org/10.1021/j100212a039

54. Zheng-Hua Ye, Vascular tissue differentiation and pattern formation in plants. In: Annual review of plant biology 53.1 (2002) 183-202. https://doi.org/10.1146/ annurev.arplant.53.100301.135245

55. Anatol M Zhabotinsky, A history of chemical oscillations and waves. In: Chaos: An Interdisciplinary Journal of Nonlinear Science 1.4 (1991) 379-386. https://doi .org/10.1063/1. 165848 